\title{
Distribution and Characteristics of Colletotrichum spp. Associated with Anthracnose of Strawberry in Hubei, China
}

Y. C. Han, X. G. Zeng, and F. Y. Xiang, Institute of Industrial Crops, Hubei Academy of Agricultural Sciences, Wuhan 430064, China; L. Ren, Oil Crops Research Institute of the Chinese Academy of Agricultural Sciences, Wuhan 430062, China; and F. Y. Chen and Y. C. Gu, Institute of Industrial Crops, Hubei Academy of Agricultural Sciences

\begin{abstract}
Han, Y. C., Zeng, X. G., Xiang, F. Y., Ren, L., Chen, F. Y., and Gu, Y. C. 2016. Distribution and characteristics of Colletotrichum spp. associated with anthracnose of strawberry in Hubei, China. Plant Dis. 100:996-1006.

Anthracnose caused by Colletotrichum spp. is a serious disease of strawberry. The etiology of anthracnose of strawberry is complex, and several Colletotrichum spp. have been regarded as causal agents. In the present study, multilocus (actin, $\beta$-tubulin, calmodulin, glyceraldehyde-3phosphate dehydrogenase, and chitin synthase) phylogenetic analysis revealed that 100 isolates of Colletotrichum associated with anthracnose of strawberry in central China belong to five species. In total, 97 isolates were identified belonging to the Colletotrichum gloeosporioides species complex, with $C$. murrayae, C. gloeosporioides, C. fructicola, and C. aenigma accounting for $81,8,4$, and $4 \%$ of the total isolates, respectively. Three isolates belonging to the $C$. acutatum complex were identified as $C$. nymphaeae. On inoculated strawberry plants, isolates of C. fructicola and C. murrayae species showed strong pathogenicity to both leaves and petioles of strawberry, with plant mortality 30 days after

inoculation of 77.8 and $55.6 \%$, respectively. C. gloeosporioides, C. aenigma, and $C$. nymphaeae showed strong pathogenicity to leaves but weak pathogenicity to petioles, with plant mortality 30 days after inoculation of 5.6, 16.7 , and $11.1 \%$, respectively. The five species were divided into four classes based on their maximum growth temperatures. Isolates of C. murrayae and $C$. gloeosporioides were more tolerant to high temperature $\left(>34^{\circ} \mathrm{C}\right)$ than isolates of other species, followed by $C$. fructicola and $C$. aenigma. Isolates of $C$. nymphaeae, which are only distributed in areas of higher altitude $(1,100 \mathrm{~m})$, were highly sensitive to higher temperature. These results indicate that pathogenicity and adaptation to temperature are important factors in the distribution of Colletotrichum spp. on strawberry plants. This research may increase our understanding of how Colletotrichum spp. emerge and spread to geographical regions with different latitudes or elevations.
\end{abstract}

Strawberry (Fragaria $\times$ ananassa) is a small fruit crop of global importance. The area used for strawberry cultivation in China has increased rapidly in recent years. Anthracnose can cause considerable yield losses under appropriate environmental conditions (Henz et al. 1992). Strawberry infected with Colletotrichum spp. may develop lesions on the petiole and crown, in serious cases resulting in the collapse and death of the plant. In nurseries, lesions form on stolons, resulting in the wilting and death of unrooted daughter plants (Freeman and Katan 1997). Infected transplants are capable of spreading the disease from the nursery to the field, where typical anthracnose symptoms are later manifested as irregular and black leaf spots, crown rot, flower blight, and fruit rot (Howard et al. 1992). Three species have been reported as causal agents of strawberry anthracnose: Colletotrichum gloeosporioides, C. fragariae, and C. acutatum (Denoyes-Rothan et al. 2003).

Anthracnose disease caused by Colletotrichum spp. affects a wide variety of woody and herbaceous plants, particularly tropical and subtropical crops and fruit trees (Cannon et al. 2012; Freeman et al. 1998; Hyde et al. 2009; Sutton 1992; Wikee et al. 2011). The genus was recently denoted the world's eighth most important group of plant-pathogenic fungi, based on perceived scientific and economic importance (Dean et al. 2012). However, the identification of Colletotrichum spp. has long been problematic. Single-gene phylogenetic analyses have not been successful in delineating species (Hyde et al. 2009). Ko Ko et al. (2011) noted the need to resurvey plant pathogens such as Colletotrichum spp. using molecular approaches because previous data based on morphology and internal transcribed spacer sequences are likely incorrect. Understanding of Colletotrichum spp. has been significantly improved with the use

Corresponding author: Y. C. Gu; E-mail: gych119@126.com

Accepted for publication 14 December 2015.

http://dx.doi.org/10.1094/PDIS-09-15-1016-RE

(C) 2016 The American Phytopathological Society of multilocus phylogenetic analysis on species complexes, as well as the epitypification of many important pathogenic species (Cai et al. 2009; Cannon et al. 2012; Damm et al. 2012a; Doyle et al. 2013; Su et al. 2011; Weir et al. 2012). An important contribution to the taxonomy of the $C$. gloeosporioides and $C$. acutatum species complex was made by Weir et al. (2012) and Damm et al. (2012a). Hyde et al. (2014) reviewed the species identification of members of Colletotrichum and revealed that the C. gloeosporioides complex consisted of 34 taxa. The $C$. acutatum complex currently comprises at least 31 taxa (Damm et al. 2012a).

Hitherto, little was known about the species and distribution of Colletotrichum associated with strawberry anthracnose in China. The first objective of this study was to identify and characterize the species associated with strawberry anthracnose in central China based on morphological, molecular, and pathogenic characteristics. The second objective was to analyze the distribution of Colletotrichum spp. based on a large number of isolates.

\section{Material and Methods}

Fungal isolate collections. Hubei Province is in the central region of the Yangtze River and has a warm, humid, subtropical climate (Zhang et al. 2009). From 2012 to 2014, plants exhibiting stunting, chlorosis, and root necrosis were collected from nurseries and production fields in central China. Thirteen locations were sampled from different geographical regions in central China. The altitudes of the sampling locations were determined by GPS positioning using an HTC desire 816D (HTC Co., Ltd., Shanghai, China). Most areas of Hubei Province are plains area with an elevation of no more than $100 \mathrm{~m}$. The 10-day average temperatures from 2012 to 2013 in Wuhan and Lichuan were supplied by the meteorological service center of Hubei Province, China. The 10-day average temperature in Wuhan (with an elevation of less than $100 \mathrm{~m}$ ) from July to September was 20.7 to $32.4^{\circ} \mathrm{C}$ in 2012 and 2013 (Fig. 1). Enshi is a mountainous area to the west of Hubei Province; the elevation of the sampling location in Enshi was approximately 1,100 m. The 10-day average temperature from July to September in Lichuan 
(the sampling area in Enshi) was 16.1 to $25.2^{\circ} \mathrm{C}$ in 2012 and 2013 (Fig. 1).

Fungi were isolated from diseased tissues such as petioles, stolons, leaf lesions, or diseased crowns of infected strawberry plants. The diseased plant tissues were surface disinfected in $70 \%$ ethanol for $10 \mathrm{~s}$ and $1 \% \mathrm{NaClO}_{3}$ for $3 \mathrm{~min}$, then rinsed three times with sterile distilled water and, finally, dried with sterilized tissue paper. The samples were cut into 5-by-5-mm pieces and plated onto potato dextrose agar (PDA) acidified with lactic acid at a final concentration of $0.05 \%$ to inhibit bacterial growth. The isolation plates were incubated under continuous fluorescent light in an incubator at $28^{\circ} \mathrm{C}$ for 3 to 7 days. The growing edges of any fungal hyphae developing on the PDA plates were then transferred aseptically to new PDA plates. The initial identification was based on the colony morphology on PDA plates. Single-spore subcultures were obtained for Colletotrichum isolates, and isolates without conidia on the PDA plates were purified by transferring samples from the edge of each colony to new PDA plates three to five times. In total, 100 Colletotrichum isolates were obtained from typical anthracnose lesions from strawberry petioles, stolons, and leaves. Of these, 98 isolates were from Hubei Province and 2 isolates $(\mathrm{Nj}-2$ and $\mathrm{Nj}-122)$ were obtained from Jiangsu Province in October. All isolates were stored at $10^{\circ} \mathrm{C}$ for about 1 to 2 weeks before DNA extraction.

Extraction of fungal DNA. To obtain mycelia from Colletotrichum spp., a 5-mm-diameter plug from the growing margin of a 5-day-old colony was inoculated on the center of an autoclaved cellophane membrane placed on PDA $(\mathrm{pH} 7.0)$ in a petri dish $(90 \mathrm{~mm}$ in diameter) (Lou et al. 2015). The dish was incubated at $28^{\circ} \mathrm{C}$ for 4 days. The mycelia of each isolate in the dish were collected using an autoclaved spatula and stored at $-20^{\circ} \mathrm{C}$ until use. The mycelia of each isolate were ground to a fine powder in liquid nitrogen with a mortar and pestle. Genomic DNA (gDNA) was extracted and purified as described elsewhere (Lee and Taylor 1990). DNA samples from each isolate were stored at $-20^{\circ} \mathrm{C}$ until use.

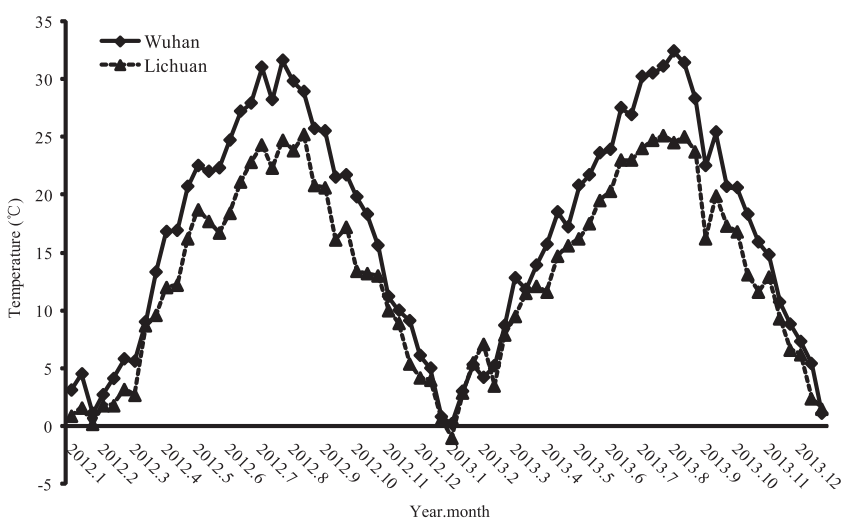

Fig. 1. The 10-day average temperature from 2012 to 2013. Data were supplied by the Hubei meteorological service center.
Polymerase chain reaction and DNA sequencing. Partial sequences corresponding to actin (ACT), $\beta$-tubulin (TUB2), calmodulin (CAL), glyceraldehyde-3-phosphate dehydrogenase (GPDH), and chitin synthase (CHS-1) were amplified from gDNA from the indicated Colletotrichum isolates (Table 1) by polymerase chain reaction (PCR). ACT and TUB2 were amplified using the primer pairs ACT512F and ACT-R2, and T1 and Tub2-R2, respectively. CAL, GPDH, and CHS-1 were amplified using the primer pairs CL1C and CL2C, GDF2 and GDR3, and CHS-88F and CHS-834R, respectively (Table 1). Primers of Act-R2, TUB-R2, GDR3, and CHS-834R were designed downstream of the binding site of standard primers (Weir et al. 2012) at these loci. All new primers designed in this study from the conserved domain in the corresponding genes in C. crassipes, C. graminicola, and C. gloeosporioides provided more specific amplification for species in this study (data not presented). The primers were used for PCR and PCR product sequencing unidirectionally, because this was more convenient than sequencing from both ends.

The PCR amplification mixtures were set up in a $0.25-\mathrm{ml}$ tube containing $60 \mu \mathrm{l}$ of reaction mixture comprising $1 \mu \mathrm{l}$ of each primer solution $(50 \mu \mathrm{M}), 2 \mu \mathrm{l}$ of dNTP mix solution $(2.5 \mathrm{mM}), 1 \mu \mathrm{l}$ of gDNA solution (approximately $50 \mathrm{ng}$ per reaction mixture), $1 \mu \mathrm{l}$ of Taq DNA polymerase (5 U per reaction mixture; TAKARA Biotechnology Co., Ltd., Dalian, China), $6 \mu$ l of PCR buffer, and $49 \mu$ l of PCR-grade water.

PCR was performed in a thermal cycler (PTC-100TM Peltier Thermal Cycler; Hercules, CA). The cycling parameters for GPDH consisted of a denaturation step at $94^{\circ} \mathrm{C}$ for $5 \mathrm{~min}$; followed by 30 cycles of $94^{\circ} \mathrm{C}$ for $30 \mathrm{~s}, 65^{\circ} \mathrm{C}$ for $30 \mathrm{~s}$, and $72^{\circ} \mathrm{C}$ for $1 \mathrm{~min}$; and a final cycle at $72^{\circ} \mathrm{C}$ for $10 \mathrm{~min}$. The cycling parameters for CAL were $94^{\circ} \mathrm{C}$ for $5 \mathrm{~min}$; followed by 30 cycles of $94^{\circ} \mathrm{C}$ for $1 \mathrm{~min}, 55^{\circ} \mathrm{C}$ for $30 \mathrm{~s}$, and $72^{\circ} \mathrm{C}$ for $2 \mathrm{~min}$; and a final step at $72^{\circ} \mathrm{C}$ for $10 \mathrm{~min}$. The cycling parameters for the ACT, TUB2, and CHS-1 regions consisted of a 5-min denaturing step at $94^{\circ} \mathrm{C}$; followed by 30 cycles of $94^{\circ} \mathrm{C}$ for $30 \mathrm{~s}, 59^{\circ} \mathrm{C}$ for $30 \mathrm{~s}$, and $72^{\circ} \mathrm{C}$ for $1 \mathrm{~min}$; and a final cycle of $10 \mathrm{~min}$ at $72^{\circ} \mathrm{C}$. A 5 - $\mu \mathrm{l}$ aliquot of each PCR was separated by electrophoresis in a $1.5 \%$ agarose gel in $1.0 \times$ Tris-acetate EDTA buffer and photographed under UV light after staining with Gel Red for $30 \mathrm{~min}$. PCR products containing the target band were purified using the AxyPrep PCR Cleanup Kit (Axygen Biological Technology Co., Ltd., Hangzhou, Zhejiang Province, China) according to the manufacturer's instructions, then individually sequenced on an $\mathrm{ABI}$ PRISM 377-96 automatic sequencer (Beijing AuGCT Biotechnol. Co., Ltd., Beijing) via the dideoxynucleotide termination method using a Big Dye Terminator v2.0 Sequencing Kit (Applied Biosystems Inc., Foster City, CA).

All sequences were divided into 25 groups by genes and species (based on the result of preliminary phylogenetic analyses) and were aligned with DNAMAN (v. 7.0). Sequences with single-nucleotide polymorphisms were amplified and sequenced a total of three times to ensure that polymorphisms were not due to sequencing errors.

Molecular identification and phylogenetic analyses. The partial DNA sequences of ACT, CAL, CHS-1, GPDH, and TUB2 for all isolates, except Lch-5, Lch-9-1, and Lch-13 (Table 2), representative

Table1. Primers used in this study

\begin{tabular}{lllcll}
\hline Gene $^{\mathbf{y}}$ & Primers & Direction & Length $(\mathbf{b p})$ & \multicolumn{1}{c}{ Sequence } & \multicolumn{1}{c}{ Reference } \\
\hline$A C T$ & ACT-512F & Forward & 399 & ATGTGCAAGGCCGGTTTCGC & Carbone and Kohn (1999) \\
& ACT-R2 & Reverse & $\ldots$ & AGGTGTGGTGCCAAATCTTCTC & This study \\
$T U B 2$ & T1 & Forward & 870 & AACATGCGTGAGATTGTAAGT & O'Donnell and Cigelnik (1997) \\
& TUB2-R2 $2^{z}$ & Reverse & $\ldots$ & TGGGTGATCTGGAAACCCTG & This study \\
CAL & CL1C & Forward & 773 & GAATTCAAGGAGGCCTTCTC & Weir et al. (2012) \\
& CL2C & Reverse & $\ldots$ & CTTCTGCATCATGAGCTGGAC & Weir et al. (2012) \\
GAPDH & GDF2 & Forward & 870 & TCCCATCAAGGTCGGCATCA & This study \\
& GDR3 & Reverse & $\ldots$ & ACCTTGCCGACAGCCTTGG & This study \\
CHS-1 & CHS-88F & Forward & 779 & GATGCCTGGAAGAAGATTGTCGT & This study \\
& CHS-834R & Reverse & $\ldots$ & GTCTCGCCAGTAGCGGACTTGAC & This study \\
\hline
\end{tabular}

\footnotetext{
y Actin (ACT), $\beta$-tubulin (TUB2), calmodulin $(C A L)$, glyceraldehyde-3-phosphate dehydrogenase (GPDH), and chitin synthase $(C H S$-1).
}

${ }^{\mathrm{z}}$ Used for polymerase chain reaction product sequencing. 
isolates of recognized species within the $C$. gloeosporioides complex (Hyde et al. 2014; Weir et al. 2012), and isolate CBS123755 of C. boninense (Damm et al. 2012b), were used to establish a five-locus combined dataset (ACT + CAL + CHS-1 + GPDH + TUB2) in the first Bayesian inference (A) analysis. C. boninense (ICMP17958) was used as an outgroup. The sequence of CAL was missing for several representative isolates within the $C$. acutatum species complex. Therefore, partial DNA sequences of ACT, CHS-1, GADH, and TUB2 for isolates Lch-5, Lch-9-1, and Lch-13 (Table 2) and for 31 representative isolates (Damm et al.2012a; Hyde et al. 2014) were used to establish a four-locus combined dataset (ACT + CHS-1 + $\mathrm{GPDH}+\mathrm{TUB} 2)$ in the second Bayesian inference (B) analysis. C. pseudoacutatum was used as an outgroup in this analysis.

Multiple sequence alignments of each gene were constructed using ClustalW as implemented in MEGA v.5 (Tamura et al. 2011). The sequences generated for the genes in this study were manually truncated to align with the reference sequences of the type isolates for constructing phylogenetic trees. Bayesian inference was used to reconstruct the phylogenetic trees using MrBayes v. 3.2.1 (Ronquist et al. 2012). The best-fit models of nucleotide substitution for ACT

Table 2. Strains and sequence used in this study

\begin{tabular}{|c|c|c|c|c|c|c|c|c|c|}
\hline \multirow[b]{2}{*}{ Species } & \multirow[b]{2}{*}{ Isolates } & \multirow[b]{2}{*}{ Substrate } & \multirow[b]{2}{*}{ Locality } & \multirow[b]{2}{*}{ Date $^{z}$} & \multicolumn{5}{|c|}{ GenBank accession number } \\
\hline & & & & & GPDH & CHS-1 & CAL & ACT & TUB2 \\
\hline Colletotrichum aenigma & Lbwuh-4-2 & Leaf & Wuhan, Hubei, China & 2013.05 & KM053192 & KM053001 & KJ420134 & KM053098 & KJ420040 \\
\hline C. aenigma & Wha-2 & Leaf & Wuhan, Hubei, China & 2012.08 & KM053193 & KM053002 & KJ420135 & KM053099 & KJ420041 \\
\hline C. aenigma & Hzau-1 & Leaf & Wuhan, Hubei, China & 2014.10 & KP339285 & KP339292 & KP339280 & KP339273 & KP339266 \\
\hline C. aenigma & Hzau-2-1 & Leaf & Wuhan, Hubei, China & 2014.10 & KP339286 & KP339293 & KP339281 & KP339274 & KP339267 \\
\hline C. fructicola & Fch-3 & Stolons & Xiangyang, Hubei, China & 2012.08 & KM053201 & KM053010 & KJ420143 & KM053107 & KJ420049 \\
\hline C. fructicola & Sha-4 & Crown & Jingzhou, Hubei, China & 2012.12 & KM053202 & KM053011 & KJ420144 & KM053108 & KJ420050 \\
\hline C. fructicola & Sab-4-3 & Crown & Jingzhou, Hubei, China & 2013.06 & KM053203 & KM053012 & KJ420145 & KM053109 & KJ420051 \\
\hline C. fructicola & Lch-39-2 & Leaf & Enshi, Hubei, China & 2013.09 & KM053204 & KM053013 & KJ420146 & KM053110 & KJ420052 \\
\hline C. gloeosporioides & Lch-1911 & Petiole & Enshi, Hubei, China & 2013.07 & KM053194 & KM053003 & KJ420136 & KM053100 & KJ420042 \\
\hline C. gloeosporioides & Jsh-6-1 & Leaf & Wuhan, Hubei, China & 2013.07 & KM053195 & KM053004 & KJ420137 & M053101 & KJ420043 \\
\hline C. gloeosporioides & Jsh-6-2 & Leaf & Wuhan, Hubei, China & 2013.07 & KM053196 & KM053005 & KJ420138 & M053102 & KJ420044 \\
\hline C. gloeosporioides & Jsh-7-1 & Petiole & Wuhan, Hubei, China & 2013.07 & KM053197 & KM053006 & KJ420139 & KM053103 & KJ420045 \\
\hline C. gloeosporioides & Wha-4 & Petiole & Wuhan, Hubei, China & 2012.08 & KM053198 & KM053007 & KJ420140 & KM053104 & KJ420046 \\
\hline C. gloeosporioides & Yd-2 & Petiole & Yichang, Hubei, China & 2012.07 & KM053199 & KM053008 & KJ420141 & KM053105 & KJ420047 \\
\hline C. gloeosporioides & Хya-221 & Stolons & Xiangyang, Hubei, China & 2012.08 & KM053200 & KM053009 & KJ420142 & KM053106 & KJ420048 \\
\hline C. gloeosporioides & Hzau-5-2 & Leaf & Wuhan, Hubei, China & 2014.10 & KP339287 & KP339294 & KP339282 & KP339275 & KP339268 \\
\hline C. murrayae & 7hao & Petiole & Wuhan, Hubei, China & 2012.08 & KM053111 & KM052920 & KJ420053 & KM053017 & KJ419959 \\
\hline C. murrayae & S-10-1 & Petiole & Wuhan, Hubei, China & 2013.08 & KM053112 & KM052921 & KJ420054 & KM053018 & KJ419960 \\
\hline C. murrayae & $s-10-4$ & Petiole & Wuhan, Hubei, China & 2013.08 & KM053113 & KM052922 & KJ420055 & KM053019 & KJ419961 \\
\hline C. murrayae & Ds-12-1 & Petiole & Wuhan, Hubei, China & 2013.08 & KM053114 & KM052923 & KJ420056 & KM053020 & KJ419962 \\
\hline C. murrayae & Ezh-2 & Petiole & Ezhou, Hubei, China & 2012.08 & KM053115 & KM052924 & KJ420057 & KM053021 & KJ419963 \\
\hline C. murrayae & Ezh-3 & Crown & Ezhou, Hubei, China & 2012.08 & KM053116 & KM052925 & KJ420058 & M053022 & KJ419964 \\
\hline C. murrayae & Fch-2 & Stolons & Xiangyang, Hubei, China & & KM053117 & KM052926 & & M053023 & KJ419965 \\
\hline C. murrayae & Fch-3-1 & Stolons & Xiangyang, $\mathrm{H}$ & & KM & KM0 & & 024 & 966 \\
\hline C. murrayae & Gay-7-3 & Crown & Jingzhou, $\mathrm{Hu}$ & 2013.06 & KM0 & KM052 & KJ420061 & 025 & KJ4 \\
\hline C. murrayae & $y-7-4$ & Crown & Jing & 2013.06 & 3120 & KM052929 & KJ420062 & 3026 & KJ41C \\
\hline C. murrayae & Gay-9-1 & Crown & Jingzhou, Hubei, China & 2013.06 & KM053121 & KM052930 & KJ420063 & KM053027 & KJ419969 \\
\hline C. murrayae & Gsh-2 & Stolons & Suizhou, Hubei, China & 2012.08 & KM053122 & KM052931 & KJ420064 & KM053028 & KJ419970 \\
\hline C. murrayae & Hga-6-2 & Crown & Huanggang, Hubei, China & 2012.08 & KM053123 & KM052932 & KJ420065 & KM053029 & KJ419971 \\
\hline C. murrayae & $y-2-2$ & Stolons & Xianing, Hubei, China & 2013.07 & KM053124 & KM052933 & KJ420066 & KM053030 & KJ419972 \\
\hline C. murrayae & Hy-2-5 & Stolons & Xianing, Hubei, China & 2013.07 & KM053125 & KM052934 & KJ420067 & KM053031 & KJ419973 \\
\hline C. murrayae & Hy- 8 & Leaf & Xianing, Hubei, China & 2013.07 & KM053126 & KM052935 & KJ420068 & KM053032 & KJ419974 \\
\hline C. murrayae & Jy-3-2 & Leaf & Xianing, Hubei, China & 2013.07 & KM053127 & KM052936 & KJ420069 & KM053033 & KJ419975 \\
\hline C. murrayae & IC-10-1 & Stolons & Huanggang, Hubei, China & 2013.08 & KM053128 & KM052937 & KJ420070 & KM053034 & KJ419976 \\
\hline C. murrayae & MC-10-3 & Stolons & Huanggang, $\mathrm{Hu}$ & & 129 & KMC & KJ420071 & 3035 & 9977 \\
\hline C. murrayae & $c-13-1$ & Stolons & hina & 2013.08 & 130 & KM052939 & 20072 & 036 & KJ419978 \\
\hline C. murrayae & C-14-1 & Stolons & Huanggang, Hubei, China & 2013.08 & KM053131 & KM052940 & KJ420073 & KM053037 & KJ419979 \\
\hline C. murrayae & IC-14-2 & Stolons & Huanggang, Hubei, China & 2013.08 & KM053132 & KM052941 & KJ420074 & KM053038 & KJ419980 \\
\hline C. murrayae & MC-15-2 & Stolons & Huanggang, Hubei, China & 2013.08 & KM053133 & KM052942 & KJ420075 & KM053039 & KJ419981 \\
\hline C. murrayae & MC-17-1 & Stolons & Huanggang, Hubei, China & 2013.08 & KM053134 & KM052943 & KJ420076 & KM053040 & KJ419982 \\
\hline C. murrayae & [C-19-3 & Stolons & Huanggang, Hubei, China & 2013.08 & KM053135 & KM052944 & KJ420077 & KM053041 & KJ419983 \\
\hline C. murrayae & MC-20-2 & Stolons & Huanggang, Hubei, China & 2013.08 & KM053136 & KM052945 & KJ420078 & KM053042 & KJ419984 \\
\hline C. murrayae & MC-20-3 & Stolons & Huanggang, Hubei, China & 2013.08 & KM053137 & KM052946 & KJ420079 & KM053043 & KJ419985 \\
\hline C. murrayae & MC-2-1 & Petiole & Huanggang, Hubei, China & 2012.08 & KM053138 & KM052947 & KJ420080 & KM053044 & KJ419986 \\
\hline C. murrayae & Mc-21-2 & & Huanggang, Hubei, China & & KM053139 & KM052948 & KJ420081 & KM053045 & KJ419987 \\
\hline C. murrayae & MC-2-2 & Stolons & Huanggang, Hubei, China & & & & KJ420082 & KM053046 & KJ419988 \\
\hline C. murrayae & MC-221 & Stolons & Huanggang, Hubei, China & 2013.08 & KM053141 & KM052950 & KJ420083 & KM053047 & KJ419989 \\
\hline C. murrayae & MC-22-2 & Stolons & Huanggang, Hubei, China & 2013.09 & KM053142 & KM052951 & KJ420084 & KM053048 & KJ419990 \\
\hline C. murrayae & MC-23-2 & Stolons & Huanggang, Hubei, China & 2013.09 & KM053143 & KM052952 & KJ420085 & KM053049 & KJ419991 \\
\hline C. murrayae & MC-23-3 & Stolons & Huanggang, Hubei, China & 2013.09 & KM053144 & KM052953 & KJ420086 & KM053050 & KJ419992 \\
\hline C. murrayae & Mc-23-4 & Stolons & Huanggang, Hubei, China & 2013.09 & KM053145 & KM052954 & KJ420087 & $\begin{array}{l}\text { KM053051 } \\
\text { (continued or }\end{array}$ & $\begin{array}{c}\mathrm{KJ} 419993 \\
\text { next page }\end{array}$ \\
\hline
\end{tabular}

\footnotetext{
z Sample date: year and month $(01=$ January through $12=$ December $)$ collected.
} 
(K80+G), CAL (GTR+G), CHS-1 (K80+G), GPDH (HKY+I), and TUB2 (GTR+I) were selected with MrModelTest v. 2.3 (Nylander 2004) using the Akaike Information Criteria. The multiple nucleotide sequence alignments of all genes were concatenated, and analysis was performed twice on the full data set for $5 \times 10^{7}$ generations. Samples were taken from the results every 1,000 generations. The convergence of all parameters was verified using the Tracer program (Rambaut and Drummond 2007). The first $25 \%$ of generations were discarded as burn-in. The concatenated alignment and trees were submitted to TreeBASE (www.treebase.org) with a submission number of 16878 .

Isolates for biological characteristics. Based on the result of phylogenetic analyses, 21 representative Colletotrichum isolates obtained from different locations were selected for further characterization of colony and mycelial growth at different temperatures. The selected isolates included five $C$. murrayae isolates (Yd-8, Ydu- $15, \mathrm{Nj}-2$, $\mathrm{Nj-122}$, and MC-17-1), four C. fructicola isolates (Sab-4-3, Sha-4, Lch-39-2, and Fch-3), five C. gloeosporioides isolates (Lch-1911, Xya-221,Yd-2, Jsh-6-1, and Wha-4), four C. aenigma isolates (Wha-2, Lbwuh-4-2, Hzau-1, and Hzau-2-1), and three C. nymphaeae isolates (Lch-5, Lch-9-1, and Lch-13).

Morphological and cultural characterization. Morphological and cultural characterizations were performed according to the methods described by Cai et al. (2009). Mycelial discs (5 mm in diameter) were taken from areas near the colony edge of 5-day-old cultures, transferred to PDA, and incubated at $28^{\circ} \mathrm{C}$ under mixed white and near-UV fluorescent light sources under a cycle of $12 \mathrm{~h}$ of light and $12 \mathrm{~h}$ of darkness. The experiment was designed as a randomized complete block with three replicates of each isolate. Colony color, conidial dimensions, and morphology were assessed (Cai et al

Table 2. (continued from preceding page)

\begin{tabular}{|c|c|c|c|c|c|c|c|c|c|}
\hline \multirow[b]{2}{*}{ Species } & \multirow[b]{2}{*}{ Isolates } & \multirow[b]{2}{*}{ Substrate } & \multirow[b]{2}{*}{ Locality } & \multirow[b]{2}{*}{ Date $^{\mathrm{z}}$} & \multicolumn{5}{|c|}{ GenBank accession number } \\
\hline & & & & & GPDH & CHS-1 & CAL & ACT & TUB2 \\
\hline C. murrayae & MC-24-1 & Stolons & Huanggang, Hubei, China & 2013.09 & KM053146 & KM052955 & KJ420088 & KM053052 & KJ419994 \\
\hline C. murrayae & Mc-3-1 & Petiole & Huanggang, Hubei, China & 2012.08 & KM053147 & KM052956 & KJ420089 & KM053053 & KJ419995 \\
\hline C. murrayae & Mc-3-2 & Petiole & Huanggang, Hubei, China & 2012.08 & KM053148 & KM052957 & KJ420090 & KM053054 & KJ419996 \\
\hline C. murrayae & MC-4-1 & Petiole & Huanggang, Hubei, China & 2012.08 & KM053149 & KM052958 & KJ420091 & KM053055 & KJ419997 \\
\hline C. murrayae & MC-5-1 & Petiole & Huanggang, Hubei, China & 2012.08 & KM053150 & KM052959 & KJ420092 & KM053056 & KJ419998 \\
\hline C. murrayae & MC-5-3 & Petiole & Huanggang, Hubei, China & 2012.08 & KM053151 & KM052960 & KJ420093 & KM053057 & KJ419999 \\
\hline C. murrayae & MC-6-2 & Stolons & Huanggang, Hubei, China & 2012.08 & KM053152 & KM052961 & KJ420094 & KM053058 & KJ420000 \\
\hline C. murrayae & MC-9-3 & Stolons & Huanggang, Hubei, China & 2012.08 & KM053153 & KM052962 & KJ420095 & KM053059 & KJ420001 \\
\hline C. murrayae & $\mathrm{Nj}-122$ & Petiole & Nanjing, Jiangsu, China & 2013.09 & KM053154 & KM052963 & KJ420096 & KM053060 & KJ420002 \\
\hline C. murrayae & $\mathrm{Nj}-2$ & Petiole & Nanjing, Jiangsu, China & 2013.09 & KM053155 & KM052964 & KJ420097 & KM053061 & KJ420003 \\
\hline C. murrayae & Sab-552 & Stolons & Jingzhou, Hubei, China & 2013.06 & KM053156 & KM052965 & KJ420098 & KM053062 & KJ420004 \\
\hline C. murrayae & Sab-6-1 & Stolons & Jingzhou, Hubei, China & 2013.06 & KM053157 & KM052966 & KJ420099 & KM053063 & KJ420005 \\
\hline C. murrayae & Sha-1 & Crown & Jingzhou, Hubei, China & 2012.12 & KM053158 & KM052967 & KJ420100 & KM053064 & KJ420006 \\
\hline C. murrayae & Sha-2 & Crown & Jingzhou, Hubei, China & 2012.12 & KM053159 & KM052968 & KJ420101 & KM053065 & KJ420007 \\
\hline C. murrayae & Sha-3 & Crown & Jingzhou, Hubei, China & 2012.12 & KM053160 & KM052969 & KJ420102 & KM053066 & KJ420008 \\
\hline C. murrayae & Sha-5 & Crown & Jingzhou, Hubei, China & 2013.03 & KM053161 & KM052970 & KJ420103 & KM053067 & KJ420009 \\
\hline C. murrayae & Sha-6 & Crown & Jingzhou, Hubei, China & 2013.03 & KM053162 & KM052971 & KJ420104 & KM053068 & KJ420010 \\
\hline C. murrayae & Sha-7-1 & Crown & Jingzhou, Hubei, China & 2013.03 & KM053163 & KM052972 & KJ420105 & KM053069 & KJ420011 \\
\hline C. murrayae & Sha-7-2 & Crown & Jingzhou, Hubei, China & 2013.03 & KM053164 & KM052973 & KJ420106 & KM053070 & KJ420012 \\
\hline C. murrayae & Wha-7 & Petiole & Wuhan, Hubei, China & 2012.08 & KM053165 & KM052974 & KJ420107 & KM053071 & KJ420013 \\
\hline C. murrayae & Wha-8 & Petiole & Wuhan, Hubei, China & 2012.08 & KM053166 & KM052975 & KJ420108 & KM053072 & KJ420014 \\
\hline C. murrayae & Wuh-13 & Crown & Wuhan, Hubei, China & 2013.04 & KM053167 & KM052976 & KJ420109 & KM053073 & KJ420015 \\
\hline C. murrayae & Wuh-13-1 & Crown & Wuhan, Hubei, China & 2013.04 & KM053168 & KM052977 & KJ420110 & KM053074 & KJ420016 \\
\hline C. murrayae & Wuh-13-2 & Crown & Wuhan, Hubei, China & 2013.04 & KM053169 & KM052978 & KJ420111 & KM053075 & KJ420017 \\
\hline C. murrayae & $\mathrm{Xt}-1$ & Petiole & Xiantao, Hubei, China & 2012.08 & KM053170 & KM052979 & KJ420112 & KM053076 & KJ420018 \\
\hline C. murrayae & Xt-18-1 & Crown & Xiantao, Hubei, China & 2013.06 & KM053171 & KM052980 & KJ420113 & KM053077 & KJ420019 \\
\hline C. murrayae & Ych-1-2 & Crown & Xiaogan, Hubei, China & 2012.08 & KM053172 & KM052981 & KJ420114 & KM053078 & KJ420020 \\
\hline C. murrayae & Yd-3 & Leaf & Yichang, Hubei, China & 2012.07 & KM053173 & KM052982 & KJ420115 & KM053079 & KJ420021 \\
\hline C. murrayae & Yd-8 & Petiole & Yichang, Hubei, China & 2012.07 & KM053174 & KM052983 & KJ420116 & KM053080 & KJ420022 \\
\hline C. murrayae & Ydu-11 & Petiole & Yichang, Hubei, China & 2013.08 & KM053175 & KM052984 & KJ420117 & KM053081 & KJ420023 \\
\hline C. murrayae & Ydu-12-1 & Petiole & Yichang, Hubei, China & 2013.08 & KM053176 & KM052985 & KJ420118 & KM053082 & KJ420024 \\
\hline C. murrayae & Ydu-12-2 & Petiole & Yichang, Hubei, China & 2013.08 & KM053177 & KM052986 & KJ420119 & KM053083 & KJ420025 \\
\hline C. murrayae & Ydu-14-1 & Petiole & Yichang, Hubei, China & 2013.08 & KM053178 & KM052987 & KJ420120 & KM053084 & KJ420026 \\
\hline C. murrayae & Ydu-15 & Petiole & Yichang, Hubei, China & 2013.08 & KM053179 & KM052988 & KJ420121 & KM053085 & KJ420027 \\
\hline C. murrayae & Zhd-11 & Petiole & Wuhan, Hubei, China & 2013.10 & KM053180 & KM052989 & KJ420122 & KM053086 & KJ420028 \\
\hline C. murrayae & Zhd-13-1 & Crown & Wuhan, Hubei, China & 2013.10 & KM053181 & KM052990 & KJ420123 & KM053087 & KJ420029 \\
\hline C. murrayae & Zhd-14-1 & Crown & Wuhan, Hubei, China & 2013.10 & KM053182 & KM052991 & KJ420124 & KM053088 & KJ420030 \\
\hline C. murrayae & Zhd-4 & Crown & Wuhan, Hubei, China & 2012.12 & KM053183 & KM052992 & KJ420125 & KM053089 & KJ420031 \\
\hline C. murrayae & Zhd-4-2 & Crown & Wuhan, Hubei, China & 2012.12 & KM053184 & KM052993 & KJ420 & KM053090 & KJ420032 \\
\hline C. murrayae & Zhd-6 & Crown & Wuhan, Hubei, China & 2012.12 & KM053185 & KM052994 & KJ420127 & KM053091 & KJ420033 \\
\hline C. murrayae & Zhd-8-1 & Petiole & Wuhan, Hubei, China & 2012.12 & KM053186 & KM052995 & KJ420128 & KM053092 & KJ420034 \\
\hline C. murrayae & Zhd-8-2 & Petiole & Wuhan, Hubei, China & 2012.12 & KM053187 & KM052996 & KJ420129 & KM053093 & KJ420035 \\
\hline C. murrayae & $\mathrm{Zj}-1-2$ & Leaf & Xianning, Hubei, China & 2013.07 & KM053188 & KM052997 & KJ420130 & KM053094 & KJ420036 \\
\hline C. murrayae & $\mathrm{Zj}-3-2$ & Leaf & Xianning, Hubei, China & 2013.07 & KM053189 & KM052998 & KJ420131 & KM053095 & KJ420037 \\
\hline C. murrayae & $\mathrm{Zj}-6-1$ & Stolons & Xianning, Hubei, China & 2013.07 & KM053190 & KM052999 & KJ420132 & KM053096 & KJ420038 \\
\hline C. murrayae & $\mathrm{Zj}-9-2$ & Petiole & Xianning, Hubei, China & 2013.07 & KM053191 & KM053000 & KJ420133 & KM053097 & KJ420039 \\
\hline C. nymphaeae & Lch-5 & Stolons & Enshi, Hubei, China & 2013.07 & KP339288 & KP339295 & $\ldots$ & KP339276 & KP339269 \\
\hline C. nymphaeae & Lch-9-1 & Leaf & Enshi, Hubei, China & 2013.07 & KP339289 & KP339296 & $\ldots$ & KP339277 & KP339270 \\
\hline C. nymphaeae & Lch-13 & Leaf & Enshi, Hubei, China & 2014.08 & KP339290 & KP339297 & $\ldots$ & KP339278 & KP339271 \\
\hline
\end{tabular}


2009; Peng et al. 2012; Weir et al. 2012). Conidial suspensions of each species were prepared in lactic acid from conidial masses on PDA. The shape and color of the conidia were observed, and the size of 50 conidia per isolate was measured using a microscope (BM2000; Nanjing Jiangnan Novel Optics Co., Ltd., Nanjing, China).

Effect of temperature on mycelial growth. In total, 21 isolates of the five species were evaluated to determine the effect of temperature on radial colony growth in culture. All isolates were maintained on PDA supplemented with lactic acid at $28^{\circ} \mathrm{C}$ for 5 days. Plugs of agar with a diameter of $5 \mathrm{~mm}$ were cut from the leading edge of colonies and placed in the center of petri plates containing PDA. The plates were then wrapped with parafilm and placed in an incubator $\mathrm{BPH}-$ 9272; Shanghai Yiheng Scientific Instrument Co., Ltd., Shanghai, China) at 1 of 11 temperatures $(6,10,14,18,22,26,28,30,32$, $34,36,38,40$, and $42^{\circ} \mathrm{C}\left[ \pm 0.2^{\circ} \mathrm{C}\right]$ ) in the dark. After 4 days, colony diameters were measured and used to calculate the mycelial growth rate (in millimeters per day). The experiment was designed as a randomized complete block, with three replicates of each isolate for each temperature treatment. The experiment was repeated once.

Pathogenicity assays. Not all the Colletotrichum isolates could sporulate on PDA plus $0.2 \%$ yeast extract (PDAY); therefore, one isolate of each species that could sporulate on PDAY was selected

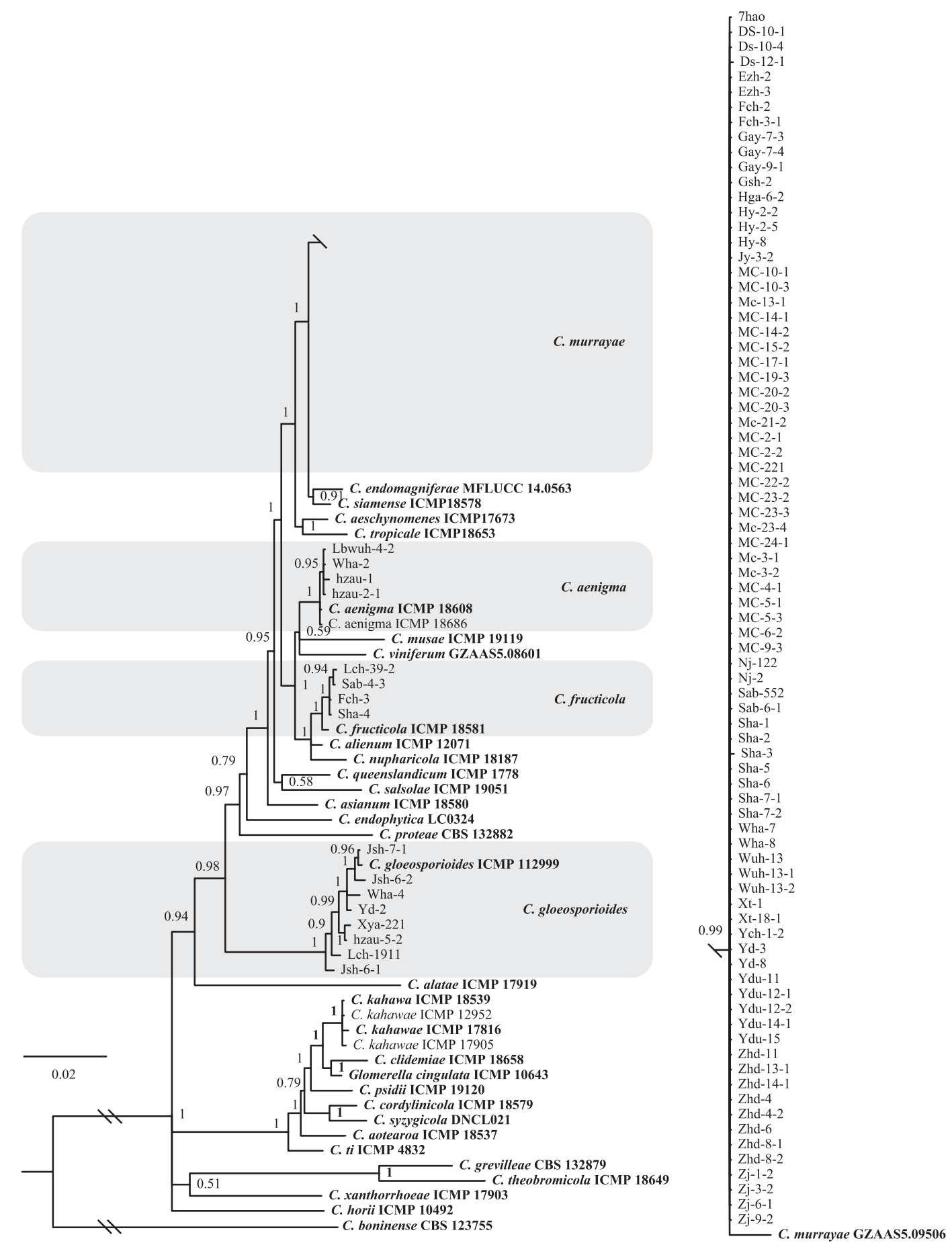

Fig. 2. Bayesian inference phylogenetic tree of 132 isolates of the Colletotrichum gloeosporioides species complex. The tree was built using concatenated sequences of the $A C T$, TUB2, CAL, GPDH, and CHS-1 genes, each with a separate model of DNA evolution. Values next to the nodes of the tree are Bayesian posterior probability values based on $5,000,000$ generations. C. boninense was used as an outgroup. Ex-type cultures are emphasized in bold, and include the taxonomic name as originally described. The scale bar indicates the number of expected changes per site. 
for pathogenicity assays. C. murrayae isolate $\mathrm{Nj}-2$, C. gloeosporioides isolate Jsh-7-1, C. fructicola isolate Lch-39-2, C. aenigma isolate Lbwuh-4-2, and C. nymphaeae isolate Lch-5 were utilized for pathogenicity tests. The five isolates were maintained on PDA supplemented with lactic acid at $28^{\circ} \mathrm{C}$ for 5 days. Plugs of agar with a diameter of $5 \mathrm{~mm}$ were cut from the leading edge of colonies and placed in the center of petri plates containing PDAY. The fungal isolates were grown at $28^{\circ} \mathrm{C}$ under mixed white and near-UV fluorescent light sources under a cycle of $12 \mathrm{~h}$ of light and $12 \mathrm{~h}$ of darkness (de los Santos Garcia de Paredes and Romero Muñoz 2002), and sporulated profusely after 7 to 10 days. Plates were flooded with sterile distilled water, the spore suspension was passed through cheesecloth to remove dislodged mycelia (Mangandi et al. 2015), and the suspension was adjusted to $2 \times 10^{6}$ conidia/ml.

Healthy 6-week-old strawberry plants ('Benihopp') devoid of any exposure to fungicides were used for plant inoculation experiments. Plants were inoculated by spraying conidial suspensions $\left(2 \times 10^{6}\right.$ conidia/ml) of the five isolates ( Nj-2, Jsh-7-1, Lch-39-2, Lbwuh-4-2, and Lch-5) until runoff (Freeman and Katan 1997). Negative control plants received sterile water without conidia. Nine plants were inoculated with each isolate separately and maintained in a moisture chamber at 25 to $35^{\circ} \mathrm{C}$ for $48 \mathrm{~h}$, then transferred to a greenhouse at 25 to $35^{\circ} \mathrm{C}$ for 30 days under natural daylight conditions.

The pathogenicity to strawberry of different species were evaluated by calculating the disease index (DI) according to the symptoms severity (SS) of leaves and petioles, respectively. The SS class of leaves, which was calculated based on a percentage of infected leaf surface caused by Colletotrichum spp., was described on a six-point scale, as follows: $0=$ healthy leaves with no lesions or $1=0.1$ to $5.0,3=5.1$ to $15.0,5=15.1$ to $30.0,7=30.1$ to 50.0 , and $9=50.1$ to $100 \%$ infected leaf surface or dead leaves. The SS class of petioles, calculated based on the length of lesions caused by Colletotrichum spp. on petioles, was described on a six-point scale, as follows: $0=$ healthy petiole with no lesions or lesions of $1=0.1$ to $2,3=2.1$ to $5.0,5=5.1$ to $9.0,7=9.1$ to 15.0 , and $9=>15.0 \mathrm{~mm}$ in length or leaves wilting) (Wang et al. 2008). All leaves and petioles on each plant were evaluated individually. The number of leaves or petioles in each SS class was recorded 20 days after inoculation (dai) and plant mortality was recorded 30 dai. These experiments were conducted twice.

SS were converted to DI that was calculated using the formula of Horiuchi and Hori (1980): DI $=\left[\left(n_{0} \times 0+n_{1} \times 1+n_{3} \times 3+n_{5} \times 5+\right.\right.$ $\left.\left.n_{7} \times 7+n_{9} \times 9\right) / 9 N\right] \times 100$, where $n$ is the number of leaves or petioles in this SS class; the subscript numbers $0,1,3,5,7$, and 9 are the SS class; and $N$ is the total number of leaves or petioles.

Data analysis. Data from the pathogenicity test were subjected to analysis of variance using the Statistical Analysis Systems (SAS, version 8.0; SAS Institute, Cary, NC) in order to determine the significance of treatment differences. Means for different species from the pathogenicity test were separated using the least significance difference (LSD) test at $P=0.05$ level. The minimum, optimum, and maximum growth temperature data were analyzed using the general linear model procedure (PROC GLM) in SAS. Species were considered fixed effects and isolates were considered random effects. When

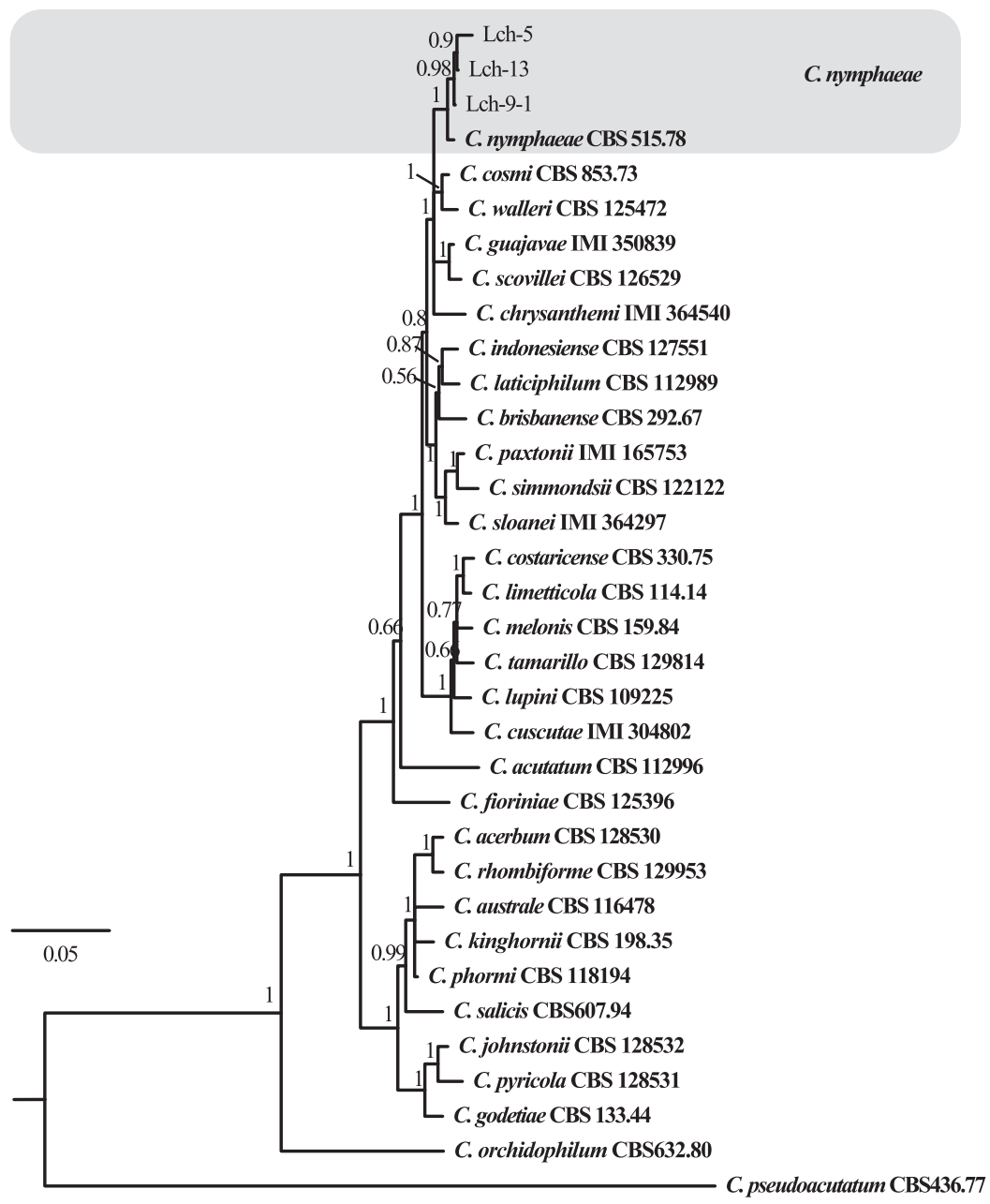

Fig. 3. Bayesian inference phylogenetic tree of 34 isolates of the Colletotrichum acutatum species complex. The tree was built using concatenated sequences of the $A C T$, TUB2, GPDH, and CHS-1 genes, each with a separate model of DNA evolution. Values next to the nodes of the tree are Bayesian posterior probability values based on 500,000 generations. C. pseudoacutatum was used as an outgroup. Ex-type cultures are emphasized in bold, and include the taxonomic name as originally described. The scale bar indicates the number of expected changes per site. 
$F$ values were significant $(P<0.05)$, species means were compared using the LSD test.

\section{Results}

Molecular identification and phylogenetic analyses. ACT, CAL, CHS-1, GPDH, and TUB2 regions of all isolates were successfully amplified and sequenced. Among the 100 isolates, 97 isolates were identified belonging to the $C$. gloeosporioides species complex and 3 isolates were identified belonging to the $C$. acutatum species complex.

The Bayesian inference $(\mathrm{A})$ with the haplotypes belonging to the C. gloeosporioides species complex was performed with the combined TUB2, ACT, CAL, GPDH, and CHS-1 sequences and comprised 2,352 characters after alignment. The boundaries of the loci used in the alignment were as follows: TUB2, 1 to 733; ACT, 734 to 1,028; CAL, 1,029 to 1,776; GPDH, 1,777 to 2,081; and CHS-1, 2,082 to 2,352 . The Bayesian inference (B) with the haplotypes belonging to the $C$. acutatum species complex was performed with the combined TUB2, ACT, GPDH, and CHS-1 sequences and comprised 1,265 characters after alignment. The boundaries of the loci used in the alignment were as follows: TUB2, 1 to 495; ACT, 496 to 745 ; GPDH, 746 to 1,015 ; CHS-1, 1,016 to 1,265 .

The isolates from strawberry belonged to five clades. In Bayesian inference (A), 81 isolates clustered with C. murrayae Li J. Peng \& K. D. Hyde; 8 isolates clustered with C. gloeosporioides (Penz.) Penz. \& Sacc., 4 isolates clustered with C. fructicola Prihastuti, L. Cai \& K. D. Hyde; and 4 isolates clustered with C. aenigma B. S. Weir \& P. R. Johnst. (Fig. 2). In Bayesian inference (B), three isolates clustered with C. nymphaeae (Pass.) Aa (Fig. 3). C. murrayae was the most frequently isolated species, comprising $81 \%$ of all isolates, followed by C. gloeosporioides at $7 \%$. Isolates of $C$. murrayae were widely distributed in Hubei Province. C. gloeosporioides was primarily distributed in Wuhan, Xiangyang, Yichang, and Enshi. C. fructicola was mainly distributed in Xiangyang, Jingzhou, and Enshi. All four isolates of C. aenigma were isolated from Wuhan. C. nymphaeae was distributed in Enshi (Fig. 4).The altitudes of the sampling locations were no higher than $100 \mathrm{~m}$, with the exception of Enshi (with an altitude of 1,100 m).

Morphological and cultural characterization. Few differences of colony morphology were observed among the five species identified when grown on PDA. All selected isolates developed white colonies that became gray after 10 days. Isolates of $C$. nymphaeae and C. gloeosporioides produced numerous conidia on PDA, while isolates of the other three species produced fewer conidia (Fig. 5). The conidia produced by $C$. nymphaeae were sharp at both ends (Fig. 6). This morphology was different from that of the other four species in this study (Table 3).

Effect of temperature on mycelial growth. The optimum temperature of all five Colletotrichum spp. in the current study was $28^{\circ} \mathrm{C}$ but the tolerance to high temperature $\left(34^{\circ} \mathrm{C}\right)$ among species was different (Fig. 7). The five species were divided into four groups based on their maximum growth temperatures (Table 4). Isolates of C. nymphaeae, which were isolated only in Enshi, were sensitive to high temperatures. The average growth rates of the three isolates were $0.11 \mathrm{~mm} /$ day at $34^{\circ} \mathrm{C}$, and no mycelial growth in vitro was observed at temperatures above $34^{\circ} \mathrm{C}$ (Table 4).

Isolates of $C$. fructicola and $C$. aenigma were similar in their tolerances to high temperatures. These two species were moderately sensitive to high temperature and grew very slowly at $36^{\circ} \mathrm{C}$, with colony diameters smaller than those of isolates of $C$. murrayae and C. gloeosporioides but larger than those of isolates of $C$. nymphaeae. No mycelial growth in vitro of the $C$. fructicola and $C$. aenigma isolates was observed at temperatures above $36^{\circ} \mathrm{C}$ (Fig. 7). Isolates of $C$. gloeosporioides and C. murrayae were tolerant to high temperatures, with a significantly larger colony diameter than the other four species when the ambient temperature was above $36^{\circ} \mathrm{C}$. Mycelial growth in vitro was observed even at $40^{\circ} \mathrm{C}$, with average growth rates of 0.11 and $0.08 \mathrm{~mm} /$ day, respectively (Fig. 7).

Pathogenicity assays. All isolates of Colletotrichum tested were pathogenic to nonwounded strawberry leaves and petioles. Symptoms first appeared on leaves. Infected leaves developed sunken, prominent, dark-brown to black decay, and necrotic lesions, and typical orange conidial masses were often observed on leaves 7 to 10 dai. Symptoms of anthracnose were not observed on petioles at 10 dai. At 20 dai, symptoms appeared on both leaves and petioles of strawberry (Fig. 5). Disease severity caused by the five species showed significant differences. Isolates of $C$. fructicola and $C$. murrayae species showed strong pathogenicity on both leaves and petioles of strawberry (Table 5). Plant mortalities caused by C. fructicola and

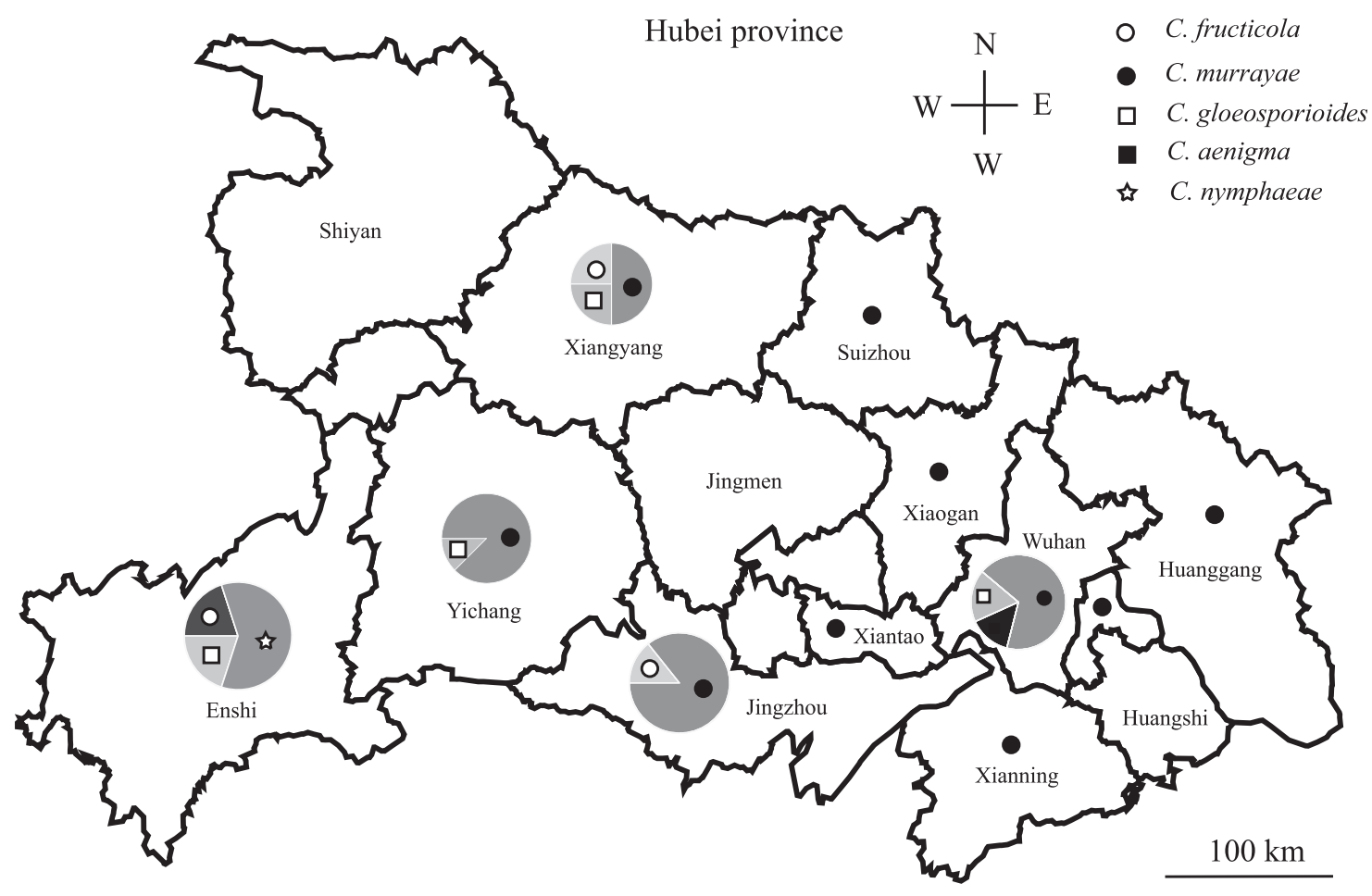

Fig. 4. Distribution of Colletotrichum spp. associated with anthracnose of strawberry in Hubei Province, China. 
C. murrayae at 30 dai were 77.8 and $55.6 \%$ respectively (Table 5). C. gloeosporioides, C. aenigma, and C. nymphaeae showed strong pathogenicity to leaves but weak to petioles. Plant mortalities caused by these species at 30 dai were 5.6, 16.7, and $11.1 \%$, respectively (Table 5), as compared with $0 \%$ for the water control plants.

\section{Discussion}

This study represents the first attempt to characterize Colletotrichum spp. associated with anthracnose of strawberry in China using a polyphasic approach. GPDH, CAL, CHS-1, ACT, and TUB2 loci were employed in the phylogenetic analysis. Phylogenetic analysis showed that the 100 Colletotrichum isolates from strawberry plants belonged to five species. This study provides the first report of C. gloeosporioides, C. aenigma, C. murrayae, C. fructicola, and C. nymphaeae from strawberry in China, while $C$. fructicola and C. nymphaeae have been recorded from strawberry previously (Damm et al. 2012a; Weir et al. 2012). Together with the 9 species in the C. gloeosporioides and C. acutatum species complexes (Damm et al. 2012a; Weir et al. 2012), 12 Colletotrichum spp. are now known to be associated with strawberry hosts. C. gloeosporioides, which was previously recognized as a pathogen of strawberry, is actually a species complex. C. gloeosporioides was typified by Cannon et al. (2008) and a recent study indicated this species was associated with the genera Citrus, Ficus, Mangifera, Pueraria, and Vitis (Weir et al. 2012). These results are in agreement with previous reports that $C$. gloeosporioides species complex is the most prevalent agent of strawberry anthracnose in China, while $C$. acutatum species complex only occasionally causes anthracnose (Xie et al. 2010). C. theobromicola (synonymous with $C$. fragariae) has been reported to infect strawberry plants (Brooks 1931; Xie et al. 2010) but no isolates of this species were identified in this study.

Important progress on taxonomy of the $C$. gloeosporioides and $C$. acutatum species complex have been made by Weir et al. (2012) and Damm et al. (2012a) but the biological characteristics such as temperature characteristics, host range, and pathogenicity of different Colletotrichum spp. is unclear. Many pathogens are sensitive to temperature, rainfall, and humidity (Harvell et al. 2002; Wilson et al. 1990). Temperature is a major factor affecting epidemics of plant disease; it can affect spore germination and infection, latent period, sporulation, spore survival, and host resistance to many pathogens (Bonde et al. 2012; Wilson et al. 1990). Temperature highs and their frequency can be factors in the delay or absence of plant disease (Bonde et al. 2012, 2013). On the other hand, the adaptation of pathogens to temperature is an important factor for disease emergence
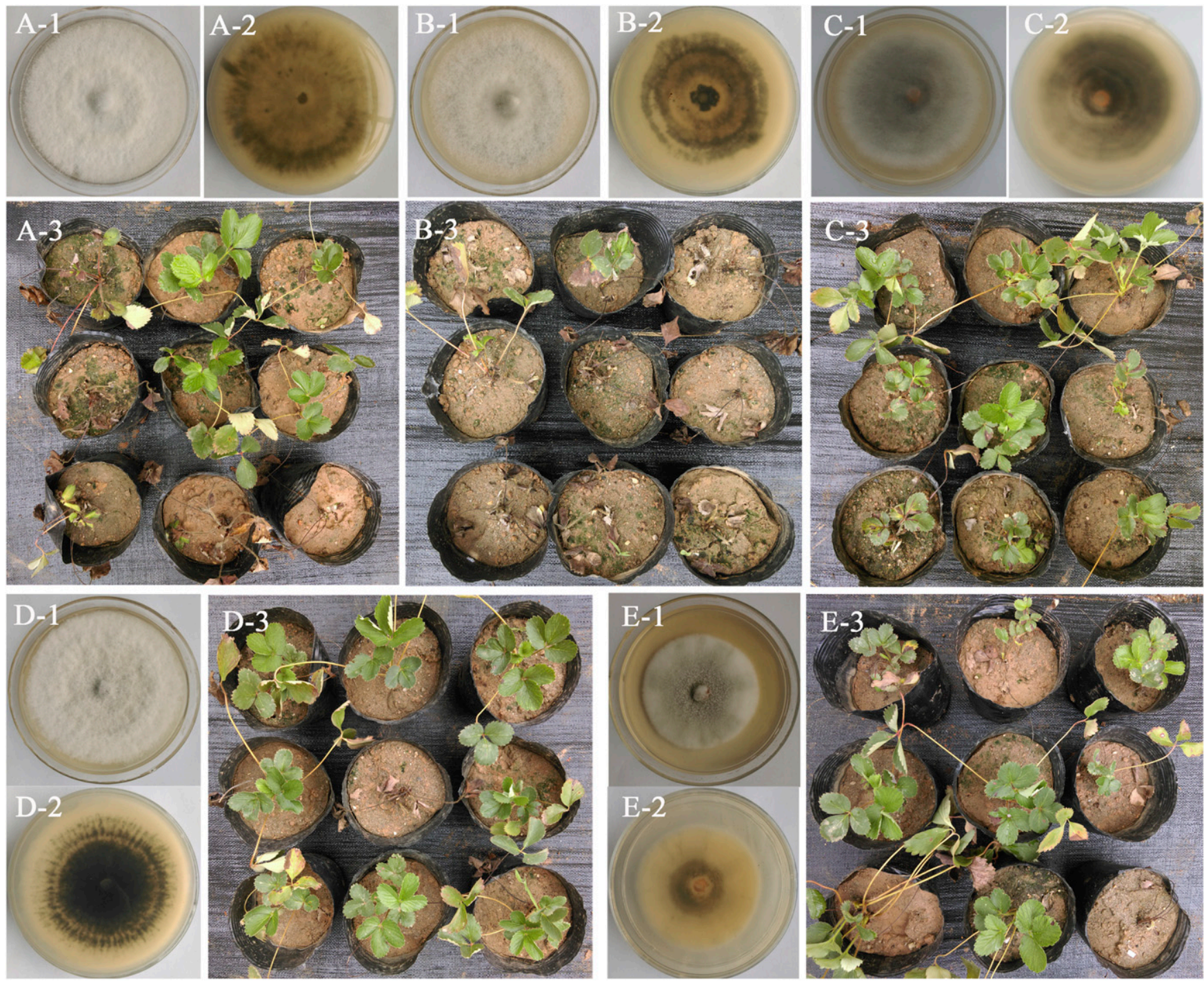

Fig. 5. Culture characteristics and pathogenicity of five Colletotrichum spp. from strawberry. A, Colletotrichum murrayae; B, C. fructicola; C, C. gloeosporioides; D, C. aenigma; and E, C. nymphaeae. Cultures on potato dextrose agar, growth at $18^{\circ} \mathrm{C}$ for 10 days from mycelial discs ( $5 \mathrm{~mm}$ in diameter) from 1 , aerial and 2 , reverse view; 3 , symptoms caused by Colletotrichum spp. on strawberry 30 days after spray inoculation with conidia. 
and spread. For example, Botrytis fruit rot of strawberry caused by Botrytis cinerea occurs mainly at low temperatures in winter and spring (Elad et al. 2007); however, Colletotrichum spp. require relatively hot temperatures and mainly cause anthracnose of strawberry in summer (Henz et al. 1992; Xie et al. 2010). The minimum, optimum, and maximum growth temperatures are important biological characteristics and widely used to characterize microorganisms (Damm et al. 2012a,b; Prihastuti et al. 2009; Weir et al. 2012). In this study, no significant differences were observed in the minimum and optimum growth temperatures of the five Colletotrichum spp. but the maximum growth temperatures were different (Table 4). The five Colletotrichum spp. were grouped into four classes based on their maximum growth temperatures.

C. murrayae was originally reported from Citrus leaves in China (Peng et al. 2012). C. murrayae is tolerant to high temperature and showed strong pathogenicity to strawberry plants. C. murrayae was the most frequently isolated species, distributed in nearly all sampling areas, and seems to be the most economically important species in strawberry nurseries in Hubei, China. C. gloeosporioides was tolerant to high temperature but was weakly pathogenic to strawberry plants. C. gloeosporioides was the second most prevalent species, with $8 \%$ of all isolates (Fig. 4). C. fructicola was originally reported causing coffee berry disease in Thailand (Prihastuti et al. 2009). Although $C$. fructicola showed stronger pathogenicity to strawberry plants than other four species in this study, it showed less tolerance to high temperature than $C$. murrayae and C. gloeosporioides, and occurred only sporadically.
C. aenigma was first described by Weir et al. (2012) and causes anthracnose of Pyrus pyrifolia in Japan and Persea americana in Israel (Weir et al. 2012). C. nymphaeae was originally described in detail in morphological and pathological terms by van der Aa (1978). A description in morphological and multilocus phylogenetic analysis of this species was provided by Damm et al. (2012a). $C$. aenigma and $C$. nymphaeae showed weak pathogenicity to strawberry plants and were less tolerant to high temperature than C. murrayae and C. gloeosporioides. C. aenigma and C. nymphaeae occurred very sporadically in Hubei, China (Fig. 4).

According to current data, the results revealed that each species possesses a stable and distinguishable maximum growth temperature, which may be used for distinguishing Colletotrichum spp., particularly morphologically similar species. The frequencies and distribution of these five species are related to differences in high temperature tolerance and pathogenicity. This research may increase our understanding of how Colletotrichum spp. emerge and spread to geographical regions with different latitudes or elevations. Due to climate change, pathogens are exposed to increasingly warmer environments, and the impact of anthropogenic climate change on terrestrial organisms is often predicted to increase with latitude and elevation (Colwell et al. 2008; Deutsch et al. 2008; Harvell et al. 2002). This research is also important for predicting changes that may occur due to global warming conditions. Further studies should be conducted on the host range, distribution, and pathogenicity of Colletotrichum spp. at different temperatures.

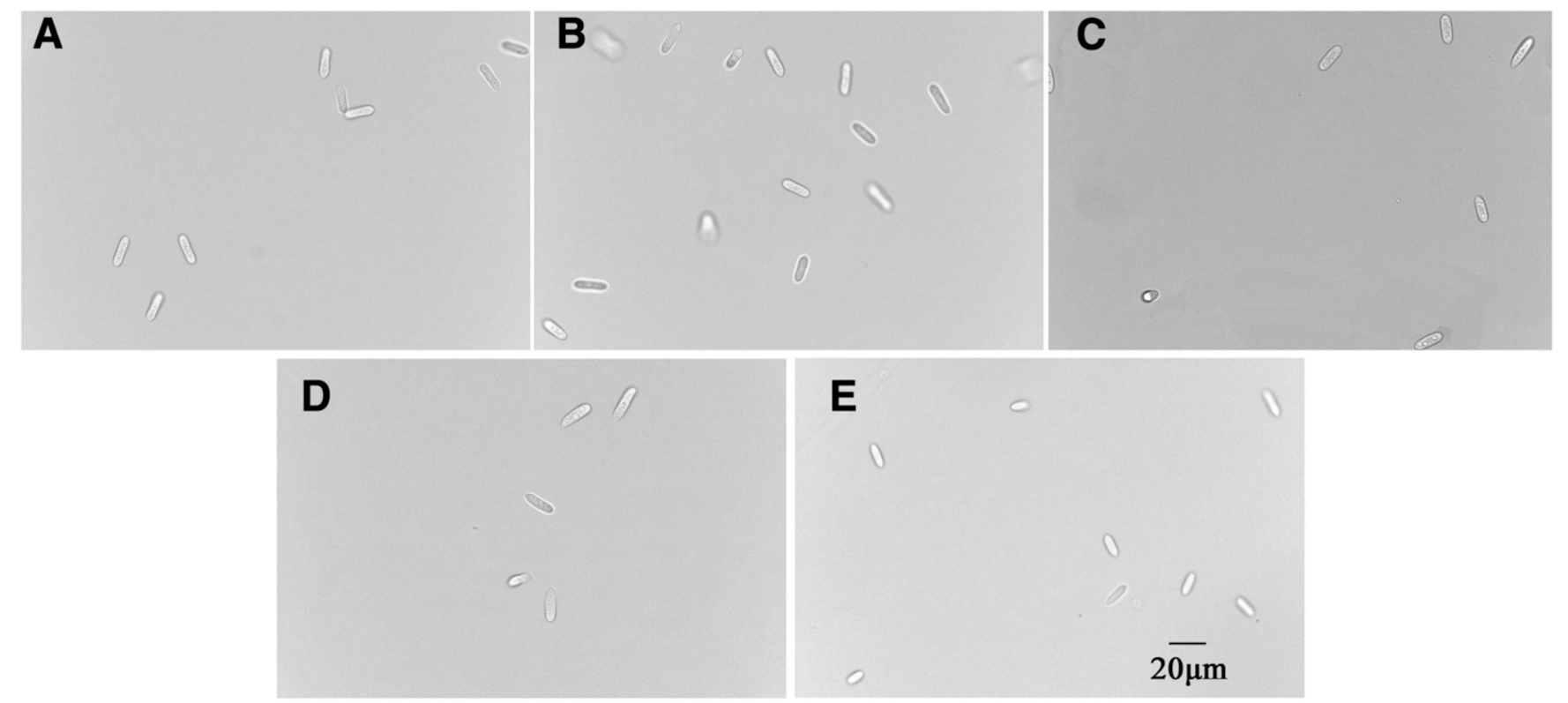

Fig. 6. Conidia of five Colletotrichum spp. from strawberry. A, Colletotrichum murrayae; B, C. fructicola; C, C. gloeosporioides; D, C. aenigma; and E, C. nymphaeae. Conidial suspensions of each species were prepared in lactic acid from conidial masses on potato dextrose agar.

Table 3. Summary of morphological data of Colletotrichum isolates

\begin{tabular}{|c|c|c|c|c|}
\hline \multirow[b]{2}{*}{ Species } & \multirow[b]{2}{*}{ Characteristics $^{\mathrm{y}}$} & \multicolumn{3}{|c|}{ Conidia $^{x}$} \\
\hline & & Length $(\mu \mathrm{m})$ & Width $(\mu \mathrm{m})$ & Shape $^{\mathrm{z}}$ \\
\hline Colletotrichum murrayae & White, lacking & $14.3 \pm 0.9(10.4-17.0)$ & $3.8 \pm 0.8(2.8-6.6)$ & Rounded \\
\hline C. gloeosporioides & White, with & $14.4 \pm 1.5(11.3-18.0)$ & $5.1 \pm 1.0(2.8-6.6)$ & Rounded \\
\hline C. fructicola & White, lacking & $14.0 \pm 0.8(11.3-15.1)$ & $4.8 \pm 0.8(2.8-6.6)$ & Rounded \\
\hline C. aenigma & White, lacking & $14.4 \pm 0.9(12.2-16.0)$ & $4.1 \pm 0.3(3.8-4.7)$ & Rounded \\
\hline C. nymphaeae & White, with & $11.2 \pm 0.8(9.4-13.2)$ & $3.9 \pm 0.5(2.8-4.7)$ & Pointed \\
\hline
\end{tabular}

$\mathrm{x}$ Data are mean \pm standard deviation or standard error, with ranges in parentheses.

y Colony characteristics: White, lacking $=$ white mycelia lacked conidial masses and White, with $=$ white to gray mycelia with mass conidial masses.

${ }^{\mathrm{z}}$ Shape at ends. 


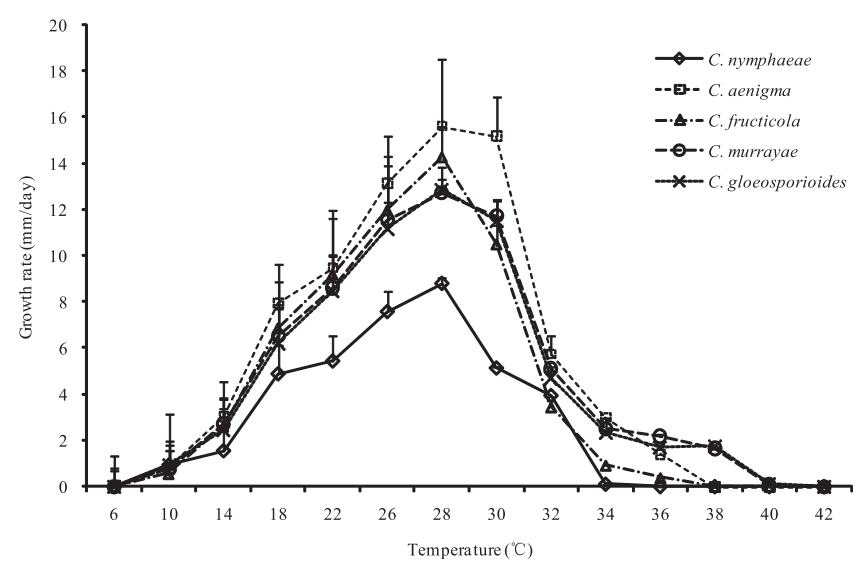

Fig. 7. Colony growth rate of Colletotrichum spp. from strawberry under different temperatures. Isolates of Colletotrichum murrayae (five isolates), C. gloeosporioides (five isolates), C. fructicola (four isolates), C. aenigma (four isolates), and C. nymphaeae (three isolates) were cultured on potato dextrose agar in the dark at different temperatures for 4 days.

Table 4. Minimum, optimum, and maximum growth temperatures of Colletotrichum spp.

\begin{tabular}{lccc}
\hline & \multicolumn{3}{c}{ Temperature $\left({ }^{\circ} \mathbf{C}\right)^{\mathbf{z}}$} \\
\cline { 2 - 4 } Species & Minimum & Optimum & Maximum \\
\hline Colletotrichum murrayae & $10.0 \mathrm{a}$ & $28.0 \mathrm{a}$ & $38.8 \mathrm{~b}$ \\
C. gloeosporioides & $10.0 \mathrm{a}$ & $28.0 \mathrm{a}$ & $40.0 \mathrm{a}$ \\
C. fructicola & $10.0 \mathrm{a}$ & $28.4 \mathrm{a}$ & $36.0 \mathrm{c}$ \\
C. aenigma & $10.0 \mathrm{a}$ & $28.0 \mathrm{a}$ & $36.0 \mathrm{c}$ \\
C. nymphaeae & $10.0 \mathrm{a}$ & $28.0 \mathrm{a}$ & $34.0 \mathrm{~d}$ \\
\hline
\end{tabular}

${ }^{\mathrm{z}}$ Mean values with the same letters are not significantly different $(P \leq 0.05)$. Isolates of C. murrayae (five isolates), C. gloeosporioides (five isolates), C. fructicola (four isolates), C. aenigma (four isolates), and C. nymphaeae (three isolates) were cultured on potato dextrose agar in the dark at different temperature for 4 days.

Table 5. Pathogenicity of Colletotrichum isolates on strawberry ${ }^{\mathrm{x}}$

\begin{tabular}{llcc}
\hline & \multicolumn{2}{c}{ Disease index $^{\mathbf{y}}$} & \\
\cline { 2 - 3 } Species & Foliar & Petiole & Mortality $(\%)^{\mathbf{z}}$ \\
\hline Colletotrichum murrayae & $70.0 \mathrm{ab}$ & $38.6 \mathrm{ab}$ & $55.6 \mathrm{~b}$ \\
C. gloeosporioides & $57.7 \mathrm{ab}$ & $4.7 \mathrm{c}$ & $5.6 \mathrm{~d}$ \\
C. fructicola & $78.5 \mathrm{a}$ & $42.4 \mathrm{a}$ & $77.8 \mathrm{a}$ \\
C. aenigma & $46.7 \mathrm{~b}$ & $8.6 \mathrm{bc}$ & $16.7 \mathrm{c}$ \\
C. nymphaeae & $53.1 \mathrm{~b}$ & $3.5 \mathrm{c}$ & $11.1 \mathrm{~cd}$ \\
LSD & 24.8 & 32.0 & 9.0 \\
\hline
\end{tabular}

${ }^{x}$ Data are mean \pm standard deviation. Mean values with the same letters were not statistically different $(P>0.05)$ according to the least significant difference (LSD) test.

y Disease index was evaluated at 20 days after inoculation.

${ }^{z}$ Percentage of plant mortality were evaluated at 30 days after inoculation. Mean percent mortality of strawberry with conidial suspension of Colletotrichum spp. by spray methods.

\section{Acknowledgments}

This study was financially supported by the Youth Science Foundation of the Hubei Academy of Agricultural Sciences (2013NKYJJ04), the Agricultural Science and Technology Innovation Center Program of Hubei Province (2007-620006-003 and 2015-620-003-001), and Open Fund Project of Hubei Key Laboratory of Crop Diseases, Insect Pests and Corp Weeds Control \& Key Laboratory of Integrated Pest Management on Crops in Central China (2015ZTSJJ8).

\section{Literature Cited}

Bonde, M. R., Nester, S. E., and Berner, D. K. 2012. Effects of daily temperature highs on development of Phakopsora pachyrhizi on soybean. Phytopathology 102:761-768.
Bonde, M. R., Nester, S. E., and Berner, D. K. 2013. Effects of frequency of "extreme" temperature highs on development of soybean rust. Phytopathology 103:708-716.

Brooks, A. N. 1931. Anthracnose of strawberry caused by Colletotrichum fragariae, n. sp. Phytopathology 21:739-744.

Cai, L., Hyde, K. D., Taylor, P. W. J., Weir, B. S., Waller, J., Abang, M. M., and Johnston, P. R. 2009. A polyphasic approach for studying Colletotrichum. Fungal Divers. 39:183-204.

Carbone, I., and Kohn, L. M. 1999. A method for designing primer sets for speciation studies in filamentous ascomycetes. Mycologia 91:553-556.

Cannon, P. F., Buddie, A. G., and Bridge, P. D. 2008. The typification of Colletotrichum gloeosporioides. Mycotaxon 104:189-204.

Cannon, P. F., Damm, U., Johnston, P. R., and Weir, B. S. 2012. ColletotrichumCurrent status and future directions. Stud. Mycol. 73:181-213.

Colwell, R. K., Brehm, G., Cardelús, C. L., Gilman, A. C., and Longino, J. T. 2008 Global warming, elevational range shifts, and lowland biotic attrition in the wet tropics. Science 322:258-261.

Damm, U., Cannon, P. F., Woudenberg, J. H. C., and Crous, P. W. 2012a. The Colletotrichum acutatum species complex. Stud. Mycol. 73:37-113.

Damm, U., Cannon, P. F., Woudenberg, J. H. C., Johnston, P. R., Weir, B. S., Tan, Y. P., Shivas, R. G., and Crous, P. W. 2012b. The Colletotrichum boninense species complex. Stud. Mycol. 73:1-36.

Dean, R., Van Kan, J. A., Pretorius, Z. A., Hammond-Kosack, K. E., Di Pietro, A., Spanu, P. D., and Foster, G. D. 2012. The top 10 fungal pathogens in molecular plant pathology. Mol. Plant Pathol. 13:414-430.

de los Santos García de Paredes, B., and Romero Muñoz, F. 2002. Effect of different fungicides in the control of Colletotrichum acutatum, causal agent of anthracnose crown rot in strawberry plant. Crop Prot. 21:11-15.

Denoyes-Rothan, B., Guérin, G., Délye, C., Smith, B., Minz, D., Maymon, M., and Freeman, S. 2003. Genetic diversity and pathogenic variability among isolates of Colletotrichum species from strawberry. Phytopathology 93: 219-228.

Deutsch, C. A., Tewksbury, J. J., Huey, R. B., Sheldon, K. S., Ghalambor, C. K., Haak, D. C., and Martin, P. R. 2008. Impacts of climate warming on terrestrial ectotherms across latitude. Proc. Natl. Acad. Sci. USA 105:6668-6672.

Doyle, V. P., Oudemans, P. V., Rehner, S. A., and Litt, A. 2013. Habitat and host indicate lineage identity in Colletotrichum gloeosporioides s.l. from wild and agricultural landscape in North America. PLoS One 8:e62394.

Elad, Y. B., Williamson, B., Tudzynski, P., and Delen, N. M., eds. 2007. Botrytis: Biology, Pathology and Control. Springer, Dordrecht, The Netherlands.

Freeman, S., and Katan, T. 1997. Identification of Colletotrichum species responsible for anthracnose and root necrosis of strawberry in Israel. Phytopathology 87:516-521.

Freeman, S., Katan, T., and Shabi, E. 1998. Characterization of Colletotrichum species responsible for anthracnose disease of various fruits. Plant Dis. 82: 596-605.

Harvell, C. D., Mitchell, C. E., Ward, J. R., Altizer, S., Dobson, A. P., Ostfeld, R. S., and Samuel, M. D. 2002. Climate warming and disease risks for terrestrial and marine biota. Science 296:2158-2162.

Henz, G. P., Boiteux, L. S., and Lopes, C. A. 1992. Outbreak of strawberry anthracnose caused by Colletotrichum acutatum in central Brazil. Plant Dis. $76: 212$.

Horiuchi, S., and Hori, M. 1980. A simple greenhouse technique for obtaining high levels of clubroot incidence. Bull. Chugoku Natl. Agric. Exp. Stn. E. 17:33-55

Howard, C. M., Maas, J. L., Chandler, C. K., and Albregts, E. E. 1992. Anthracnose of strawberry caused by the Colletotrichum complex in Florida. Plant Dis. 76:976-981.

Hyde, K. D., Cai, L., Cannon, P. F., Crouch, J. A., Crous, P. W., Damm, U., and Zhang, J. Z. 2009. Colletotrichum -names in current use. Fungal Divers. 39:147-182.

Hyde, K. D., Nilsson, R. H., Alias, S. A., Ariyawansa, H. A., Blair, J. E., Cai, L., de Cock, A. W. A. M., Dissanayake, A. H., Glockling, S. L., Goonasekara, I. D., Gorczak, M., Hahn, M., Jayawardena, R. S., van Kan, J. A. L., Laurence, M. H., Lévesque, C. A., Li, X. H., Liu, J. K., Maharachchikumbura, S. S. N., Manamgoda, D. S., Martin, F. N., McKenzie, E. H. C., McTaggart, A. R., Mortimer, P. E., Nair, P. V. R., Pawłowska, J., Rintoul, T. L., Shivas, R. G., Spies, C. F. J., Summerell, B. A., Taylor, P. W. J., Terhem, R. B., Udayanga, D., Vaghefi, N., Walther, G., Wilk, M., Wrzosek, M., Xu, J. C., Yan, J. Y., and Zhou, N. 2014. One stop shop: Backbones trees for important phytopathogenic genera: I (2014). Fungal Divers. 67:21-125.

Ko Ko, T. W., Stephenson, S. L., Bahkali, A. H., and Hyde, K. D. 2011. From morphology to molecular biology: Can we use sequence data to identify fungal endophytes? Fungal Divers. 50:113-120.

Lee, S. B., and Taylor, J. W. 1990. Isolation of DNA from fungal mycelia and single spores. Pages 282-287 in: PCR Protocols, A Guide to Methods and Applications. M. A. Innis, D. H. Gelfand, J. J. Sninsky, and T. J. White, eds. Academic Press, San Diego, CA.

Lou, Y., Han, Y. C., Yang, L., Wu, M. D., Zhang, J., Cheng, J. S., Wang, M. Y., Jiang, D. H., Chen, W. D., and Li, G. Q. 2015. CmpacC regulates mycoparasitism, oxalate degradation and antifungal activity in the mycoparasitic fungus Coniothyrium minitans. Environ. Microbiol. 17:4711-4729.

Mangandi, J., Peres, N. A., and Whitaker, V. M. 2015. Identifying resistance to crown rot caused by Colletotrichum gloeosporioides in strawberry. Plant Dis. 99:954-961. 
Nylander, J. A. A. 2004. MrModeltest v2. Program distributed by the author. Evolutionary Biology Centre, Uppsala University, Uppsala, Sweden.

O'Donnell, K., and Cigelnik, E. 1997. Two divergent intragenomic rDNA ITS2 types within a monophyletic lineage of the fungus Fusarium are nonorthologous. Mol. Phylogenet. Evol. 7:103-116.

Peng, L. J., Yang, Y. L., Hyde, K. D., Bahkali, A. H., and Liu, Z. Y. 2012. Colletotrichum species on citrus leaves in Guizhou and Yunnan provinces, China. Cryptogam., Mycol. 33:267-283.

Prihastuti, H., Cai, L., Chen, H., McKenzie, E. H. C., and Hyde, K. D. 2009. Characterisation of Colletotrichum species associated with coffee berries in northern Thailand. Fungal Divers. 39:89-109.

Rambaut, A., and Drummond, A. J. 2007. Tracer v1.4. University of Oxford, Oxfordshire, U. K. Available from: http://beast.bio.ed.ac.uk/Tracer.

Ronquist, F., Teslenko, M., Van der Mark, P., Ayres, D. L., Darling, A., Höhna, S., Larget, B., Liu, L., Suchard, M. A., and Huelsenbeck, J. P. 2012. MrBayes v. 3.2: Efficient Bayesian phylogenetic inference and model choice across a large model space. Syst. Biol. 61:539-542.

Su, Y. Y., Noireung, P., Liu, F., Hyde, K. D., Moslem, M. A., Bahkali, A. H., Abd-Elsalam, K. A., and Cai, L. 2011. Epitypification of Colletotrichum musae, the causative agent of banana anthracnose. Mycoscience 52:376-382.

Sutton, B. C. 1992. The genus Glomerella and its anamorph Colletotrichum. Pages 1-26 in: Colletotrichum: Biology, Pathology and Control. J. A. Bailey and M. J. Jeger, eds. CAB International, Wallingford, UK.
Tamura, K., Peterson, D., Peterson, N., Stecher, G., and Nei, M. 2011. MEGA5: Molecular evolutionary genetics analysis using maximum likelihood, evolutionary distance, and maximum parsimony methods. Mol. Biol. Evol. 28:2731-2739.

van der Aa, H. A. 1978. A leaf spot of Nymphaea alba in the Netherlands. Neth. J Plant Pathol. 84:109-115.

Wang, F., Ma, Y., Gao, X. Y., and Zhang, Z. H. 2008. Study on the identification techniques for determining strawberry cultivars resistance to anthracnose. J. Fruit Sci. 25:542-547.

Weir, B. S., Johnston, P. R., and Damm, U. 2012. The Colletotrichum gloeosporioides species complex. Stud. Mycol. 73:115-180.

Wikee, S., Cai, L., Pairin, N., McKenzie, E. H., Su, Y. Y., Chukeatirote, E., and Hyde, K. D. 2011. Colletotrichum species from Jasmine (Jasminum sambac). Fungal Divers. 46:171-182.

Wilson, L. L., Madden, L. V., and Ellis, M. A. 1990. Influence of temperature and wetness duration on infection of immature and mature strawberry fruit by Colletotrichum acutatum. Phytopathology 80:111-116.

Xie, L., Zhang, J. Z., Wan, Y., and Hu, D. W. 2010. Identification of Colletotrichum spp. isolated from strawberry in Zhejiang Province and Shanghai City, China. J. Zhejiang Univ. Sci. B 11:61-70.

Zhang, Z., Gong, D., Hu, M., Guo, D., He, X., and Lei, Y. 2009. Anomalous winter temperature and precipitation events in southern China. J. Geogr. Sci. 19: 471-488. 